\title{
Sarcomas de partes blandas en pacientes menores de 2 años. Serie de casos
}

\author{
Soft tissue sarcomas in infants. Case series
}

\author{
Dr. Gonzalo Magno ${ }^{a}$ Dr. José I. Albergo ${ }^{a}$, Dra. Patricia Streitenberger ${ }^{a}$, Dr. Miguel Ayerza ${ }^{a}$, \\ Dr. Germán Fafalli ${ }^{a}$ Dr. Luis Aponte-Tinao ${ }^{a}$
}

\begin{abstract}
RESUMEN
Los sarcomas de partes blandas en menores de 2 años son infrecuentes. Durante 2007-2017, 445 pacientes con sarcomas de partes blandas fueron tratados en nuestra Institución; $6(\mathrm{el} 0,5 \%)$ eran menores de 2 años. Se analizaron los resultados clínicos y oncológicos en este grupo. La edad media de diagnóstico fue 15 meses. Cuatro eran varones y 2, mujeres. El seguimiento promedio fue 29 meses. El fibrosarcoma $(n=4)$ fue la variedad más frecuente. Cinco fueron tratados con cirugía de conservación del miembro; al restante se le realizó amputación. Todos realizaron tratamiento adyuvante con quimioterapia. La supervivencia a 24 meses fue del $100 \%$. Dos pacientes presentaron recidiva local; ambos casos, antes de los 24 meses. El tratamiento quirúrgico asociado a la quimioterapia impresiona ser la mejor opción terapéutica. La proporción de recurrencia local es alta para este grupo de pacientes luego de la cirugía de conservación del miembro.

Palabrasclave:sarcomadetejidosblandos, infantes, rabdomiosarcoma, cirugía.
\end{abstract}

\begin{abstract}
Soft tissue sarcomas in children under 2 years of age are infrequent. During 2007-2017, a total of 445 patients diagnosed with soft tissue sarcomas were treated at our institution, $6(0.5 \%)$ were under 2 years. We analysed clinical and oncologic outcomes in this select group. The mean age of diagnosis was 15 months. Four patients were male and 2 female. The mean follow-up time was 29 months. Fibrosarcoma $(n=4)$ was the most frequent diagnosis. Five patients were treated with limb salvage surgery, and the remaining one had to undergo amputation. All patients received adjuvant treatment with chemotherapy. The 24-month survival rate was $100 \%$. Two patients presented a local recurrence before 24 -months followup. Surgical treatment associated with chemotherapy seems to be the best therapeutic option. Local recurrence rate after limb salvage surgery is high for this group of patients.

Key words: infants, rhabdomyosarcoma, surgery, soft tissue sarcomas.
\end{abstract}

http:/ / dx.doi.org/10.5546/ aap.2020.e162

a. Hospital Italiano de Buenos Aires, Buenos Aires, Argentina.

Correspondencia:

Dr. Gonzalo Magno: gonzalo.magno@hospitalitaliano.org.ar

Financiamiento: Ninguno.

Conflicto de intereses: Ninguno que declarar.

Recibido: 17-12-2018

Aceptado: 30-9-2019
Cómo citar: Magno G, Albergo JI, Streitenberger P, Ayerza M, et al. Sarcomas de partes blandas en pacientes menores de 2 años. Serie de casos. Arch Argent Pediatr 2020;118(2):e162-e165.

\section{INTRODUCCIÓN}

Los sarcomas de partes blandas en pacientes pediátricos representan un pequeño porcentaje de los tumores malignos de esta población. Se dividen en 2 grandes grupos: rabdomiosarcomas y no rabdomiosarcomas. ${ }^{1}$ Pueden localizarse tanto en las extremidades como en el esqueleto axial y se caracterizan por presentar mejor pronóstico que en los adultos. Pueden desarrollarse en cualquier parte del cuerpo como una masa expansiva dolorosa y estar acompañados de síntomas específicos en función de su lugar anatómico.

La mayoría de los casos descritos son en pacientes menores de 6 años; sin embargo, son infrecuentes los reportes en los menores de 2 años. ${ }^{2,3}$ La edad de presentación del tumor constituye un factor pronóstico, y es peor en los niños más jóvenes. ${ }^{4}$ Otros factores pronósticos descritos han sido la localización del tumor (extremidades vs. centrales), el grado histológico y el tipo de tumor (rabdomiosarcomas vs. no rabdomiosarcomas). ${ }^{2,5}$

El tratamiento principal en la población pediátrica de los sarcomas de partes blandas es la resección quirúrgica en bloque, con márgenes libres de tumor, asociada o no a quimioterapia. A diferencia de la población adulta, la radioterapia no suele indicarse rutinariamente debido a las potenciales complicaciones, que incluyen alteraciones del crecimiento o desarrollo de tumores secundarios. ${ }^{6}$

El objetivo de nuestro estudio fue evaluar a un grupo de pacientes menores de 2 años con sarcoma de partes blandas, así como los resultados oncológicos y clínicos, y determinar la proporción de la patología en este rango etario en nuestra Institución. 


\section{MATERIALES Y MÉTODOS}

Se realizó una búsqueda retrospectiva en nuestra base de datos oncológica entre 2007 y 2017 de todos los pacientes con diagnóstico de sarcoma de partes blandas tratados quirúrgicamente. Se incluyeron solo para el análisis pacientes menores de 2 años con diagnóstico de sarcoma de partes blandas confirmado por histología, tratados quirúrgicamente y con un seguimiento mínimo de 24 meses. No hubo pacientes con este diagnóstico que no hubieran recibido tratamiento quirúrgico en nuestra Institución.

El tratamiento de elección en todos los casos fue quirúrgico y estuvo a cargo del equipo de Ortopedia Oncológica de nuestra Institución. Todos los pacientes fueron discutidos antes en ateneo multidisciplinario y el diagnóstico inicial se realizó a través de la punción para la biopsia. Los márgenes, así como el diagnóstico final, fue definido por un patólogo especializado en Patología Oncológica Musculoesquelética.

Se analizaron todas las historias clínicas de los pacientes para la recolección de los datos demográficos y clínicos a través de un observador independiente (GM). Para el análisis de la sobrevida global, se utilizó el método de Kaplan-Meier. Se definió como el tiempo desde el diagnóstico hasta el último seguimiento o evento de muerte.

\section{RESULTADOS}

De un total de 445 pacientes con sarcomas de partes blandas tratados en nuestra Institución, 6 eran menores de 2 años y cumplían con los criterios de inclusión. La proporción de sarcomas de partes blandas en menores de 6 años fue del 0,5\%. La edad media de la serie fue de 15 meses (rango de 3 a 24 meses) y el seguimiento promedio fue de 76 meses (de 24 a 132 meses). El diagnóstico más frecuente fue fibrosarcoma $(n=4)$, seguido por rabdomiosarcoma $(n=1) y$ sarcoma sinovial $(\mathrm{n}=1)$.

\section{Resultados oncológicos}

La sobrevida de la serie fue del $100 \%$ a los 5 años. Un solo paciente presentó metástasis a distancia (fémur distal), la cual desarrolló a los 11 meses, y fue tratado con una desarticulación de cadera. Se encontraba libre de enfermedad al momento del último control (56 meses). La sobrevida libre de enfermedad de la serie fue del $92 \%$ (intervalo de confianza-IC- del $95 \%$ : 76-107) a los 12 meses y del $75 \%$ (IC $95 \%$ : 50-99) a los 24 meses.
En todos los casos, el tratamiento de elección fue quimioterapia asociada a resección quirúrgica. Cinco pacientes pudieron ser tratados con cirugías de conservación del miembro, mientras que un paciente requirió de una amputación infrapatelar por compromiso del pie y articulación tibioastragalina. Todos los pacientes cumplieron con el esquema de quimioterapia posoperatorio.

De los 5 pacientes tratados con cirugía de conservación del miembro, 3 fueron resecciones del miembro inferior y 2 del miembro superior. En 4 casos, los márgenes de resección resultaron libres de neoplasia y, en un paciente, se obtuvieron márgenes contaminados, por lo que se indicó radioterapia posoperatoria.

Dos de los 6 pacientes presentaron una recurrencia local y los casos libres de recurrencia local (ELRL) fueron, a 12 meses, el $86 \%$ (IC $95 \%$ : 54-113) y el $67 \%$ (IC $95 \%$ : 29-99) a 24 meses de la cirugía. Todas las recidivas locales se presentaron antes de los 2 años (10/13 meses) y fueron tratadas con nuevas resecciones oncológicas con márgenes libres. Los dos pacientes se encontraban libres de enfermedad al momento del último control (Figura 1).

\section{DISCUSIÓN}

Los sarcomas pediátricos de partes blandas son una patología infrecuente cuyo tratamiento difiere del de la población adulta. La mayoría de los trabajos en la literatura evalúan a la población pediátrica menor de 18 años en conjunto. Específicamente en el caso de los menores de 2 años, es una patología aún menos reportada.

Trabajos previos han demostrado que la edad constituye un factor pronóstico en los sarcomas. Es peor la expectativa en este subgrupo. No está clara la causa, ya que la biología del tumor no parece variar. Se ha propuesto que, en estos pacientes, podría administrarse una dosis de radioterapia menor, dada una mayor preocupación por los posibles efectos adversos, así como una menor tolerancia de estos pacientes a la quimioterapia., ${ }^{2,7}$

Siendo la cirugía la herramienta fundamental para el tratamiento de esta patología, su éxito depende, principalmente, de poder obtener márgenes libres, lo que disminuye la incidencia de recurrencia local. ${ }^{8}$ En nuestra serie, la cirugía fue el pilar fundamental de tratamiento, $y$, en 5, se pudo realizar una cirugía de conservación del miembro. Los márgenes son siempre un factor fundamental a la hora de las recidivas locales, y está menos comprobada su relación con respecto 
a la sobrevida. Sin embargo, es obligatorio tratar de garantizar márgenes libres de tumor. ${ }^{9}$

La radioterapia ha sido descrita como un tratamiento adyuvante efectivo para los sarcomas de partes blandas, principalmente, por su potencial de reducir el riesgo de recidiva local. ${ }^{10,11}$ Debido a los posibles efectos adversos en este grupo etario en particular, no suele utilizarse cuando los márgenes quirúrgicos son satisfactorios. ${ }^{12}$ De los 6 pacientes incluidos en el estudio, solo 1 recibió radioterapia. Posiblemente, radique ahí la explicación de nuestra alta recidiva local. La quimioterapia presenta su principal utilidad para los tumores de tipo rabdomiosarcoma, los cuales fueron una minoría en nuestra serie.

En cuanto a la localización tumoral, si bien los tumores del esqueleto axial tienen peor pronóstico y mayor recurrencia que en las extremidades, en gran parte, debido a la posibilidad de una amputación, en nuestra serie, no se han encontrado diferencias significativas entre ambas localizaciones. ${ }^{13}$ La presencia de metástasis, según la literatura, representa el factor de peor pronóstico para estos pacientes, si bien no se puede decir que haya sido el caso en nuestra serie. ${ }^{14}$
El trabajo tiene limitaciones por ser retrospectivo, con un bajo número de pacientes, heterogéneo en términos de diagnóstico y con un corto tiempo de seguimiento. Sin embargo, se trata de una patología infrecuente, poco reportada en la literatura y en un subgrupo selecto de pacientes, que resulta un desafío.

En conclusión, los sarcomas de partes blandas en menores de 2 años son infrecuentes. El tratamiento quirúrgico con márgenes libres asociado a quimioterapia impresiona ser la mejor opción terapéutica. La cirugía de conservación del miembro es posible; no obstante, el porcentaje de recurrencia local es alto. La radioterapia debe ser evaluada en cada paciente en particular.

\section{REFERENCIAS}

1. Cheung MC, Zhuge $Y$, Yang R, Ogilvie MP, et al. Incidence and outcomes of extremity soft-tissue sarcomas in children. J Surg Res. 2010; 163(2):282-9.

2. Malempati S, Rodeberg DA, Donaldson SS, Lyden ER, et al. Rhabdomyosarcoma in infants younger than 1 year: a report from the Children's Oncology Group. Cancer. 2011; 117(15):3493-501.

3. Perez EA, Kassira N, Cheung MC, Koniaris LG, et al. Rhabdomyosarcoma in children: a SEER population based study. J Surg Res. 2011; 170(2):e243-51.

4. Sultan I, Qaddoumi I, Yaser S, Rodriguez-Galindo C, et al. Comparing adult and pediatric rhabdomyosarcoma in the

FIgURA 1. Gráfico de Kaplan-Meier para la supervivencia global y la tasa de recurrencia local

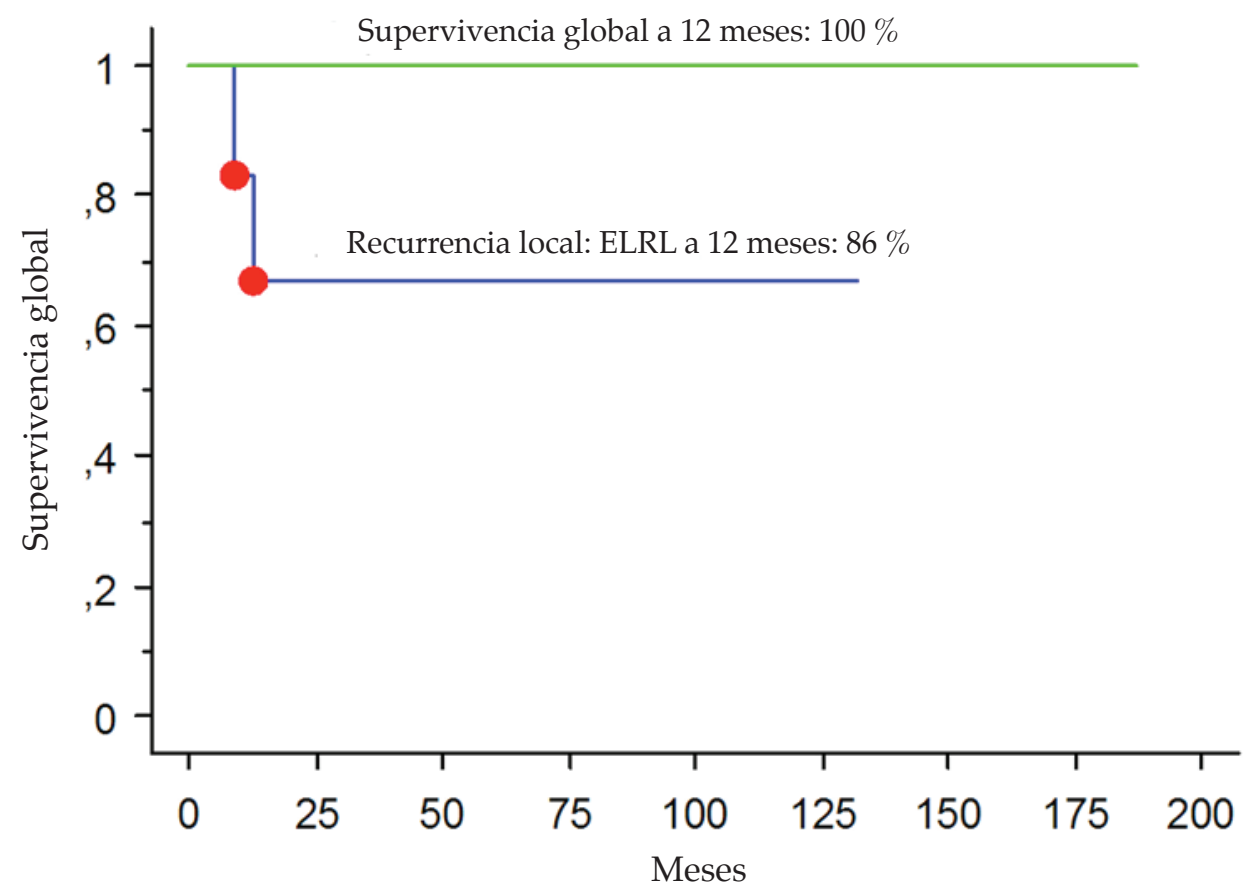

ELRL: evento libre de recurrencia local. 
surveillance, epidemiology and end results program, 1973 to 2005: an analysis of 2,600 patients. J Clin Oncol. 2009; 27(20):3391-7.

5. Guadagnolo BA, Zagars GK, Ballo MT, Strom SS, et al. Mortality after cure of soft-tissue sarcoma treated with conservation surgery and radiotherapy. Cancer. 2008; 113(2):411-8.

6. Ferrari A, Miceli R, Rey A, Oberlin O, et al. Non-metastatic unresected paediatric non-rhabdomyosarcoma soft tissue sarcomas: results of a pooled analysis from United States and European groups. Eur J Cancer. 2011; 47(5):724-31.

7. Amer KM, Thomson JE, Congiusta D, Dobitsch A, et al. Epidemiology, incidence, and survival of rhabdomyosarcoma subtypes: SEER and ICES database analysis. J Orthop Res. 2019; 37(10):2226-30.

8. Loeb DM, Thornton K, Shokek O. Pediatric soft tissue sarcomas. Surg Clin North Am. 2008; 88(3):615-27.

9. Sambri A, Bianchi G, Cucurnia I, Gambarotti M, et al. Pediatric soft tissue sarcoma of the limbs: clinical outcome of 97 patients. Eur J Orthop Surg Traumatol. 2018; 28(1):1-7.
10. Blakely ML, Spurbeck WW, Pappo AS, Pratt CB, et al. The impact of margin of resection on outcome in pediatric nonrhabdomyosarcoma soft tissue sarcoma. J Pediatr Surg. 1999; 34(5):672-5.

11. Panda SP, Chinnaswamy G, Vora T, Prasad M, et al. Diagnosis and management of rhabdomyosarcoma in children and adolescents: ICMR consensus document. Indian J Pediatr. 2017; 84(5):393-402.

12. Bradley JA, Kayton ML, Chi Y-Y, Hawkins DS, et al. Treatment approach and outcomes in infants with localized rhabdomyosarcoma: A report from the Soft Tissue Sarcoma Committee of the Children's Oncology Group. Int J Radiat Oncol Biol Phys. 2019; 103(1):19-27.

13. Spunt SL, Pratt CB, Rao BN, Pritchard M, et al. Childhood carcinoid tumors: the St Jude Children's Research Hospital experience. J Pediatr Surg. 2000; 35(9):1282-6.

14. Pappo AS, Rao BN, Jenkins JJ, Merchant T, et al. Metastatic nonrhabdomyosarcomatous soft-tissue sarcomas in children and adolescents: the St. Jude Children's Research Hospital experience. Med Pediatr Oncol. 1999; 33(2):76-82. 